\title{
ON SOME CLASSES OF MULTIVALENT STARLIKE FUNCTIONS
}

\author{
BY \\ RONALD J. LEACH
}

\begin{abstract}
Classes of multivalent functions analogous to certain classes of univalent starlike functions are defined and studied. Estimates on coefficients and distortion are made, using a variety of techniques.
\end{abstract}

1. Let St denote the class of all functions $f(z)=z+\ldots$ analytic, univalent and starlike in the unit disc $U$. Such functions satisfy the condition $\operatorname{Re}\left(z f^{\prime}(z) / f(z)\right)>0, z \in U$.

The problem of defining a corresponding class of multivalent starlike functions has been studied by several authors. Hummel [5] distinguishes six commonly used definitions, a typical one being $f(z)$ belongs to the class $S(p)$ if $f$ has at most $p$ zeros in $U$ and

$$
\limsup _{r \rightarrow 1} \min _{|z|=r} \operatorname{Re} \frac{z f^{\prime}(z)}{f(z)} \geqslant 0 .
$$

In this note we will study three classes of multivalent starlike functions which are analogues of certain subclasses of St.

2. Let $S_{1}(p, \alpha), p$ a positive integer, $0 \leqslant \alpha \leqslant 2 p$, denote the class of all functions $f(z)=a_{0}+a_{1} z+\ldots$ analytic in $U$ with precisely $p$ zeros there such that

$$
\limsup _{r \rightarrow 1} \min _{|z|=r} \operatorname{Re} \frac{z f^{\prime}(z)}{f(z)} \geqslant \alpha .
$$

$S_{1}(p, \alpha)$ is the generalization of the class $S(\alpha)$ of starlike functions of order $\alpha$ introduced by Robertson [11].

THEOREM 2.1. Let $f(z)$ belong to the class $S_{1}(p, \alpha)$ and suppose $f$ has zeros at $z_{1}, z_{2}, \ldots, z_{p}$. Then $f(z)$ is $p$-valent in $U$ and there is a function $g$ in the class $S(\alpha / p)$ and a constant $A$ such that

$$
f(z)=A \prod_{j=1}^{p} \Psi\left(z, z_{j}\right)[g(z)]^{p},
$$

Received by the editors May 6, 1974.

AMS (MOS) subject classifications (1970). Primary 30A32, $30 \mathrm{~A} 34$.

Key words and phrases. Multivalent starlike functions, coefficients, distortion theorems. 
where

$$
\Psi\left(z, z_{j}\right)=\left(z-z_{j}\right)\left(1-z_{j}^{*} z\right) / z
$$

(The asterisk denotes conjugation.)

Proof. Since inequality (2.1) implies inequality (1.1), it follows from [5] that $f(z)$ is $p$-valent and that there is a function $g \in$ St such that (2.2) holds.

We compute

$$
\frac{z f^{\prime}(z)}{f(z)}=\sum_{j=1}^{p} \frac{z \Psi^{\prime}\left(z, z_{j}\right)}{\Psi\left(z, z_{j}\right)}+p \frac{z g^{\prime}(z)}{g(z)} .
$$

Since $\operatorname{Re}\left(z \Psi^{\prime} / \Psi\right)=0$ on $|z|=1$, it follows from (2.3) and the definition of $S_{1}(p, \alpha)$ that lim sup $\min \operatorname{Re}\left(z g^{\prime}(z) / g(z)\right) \geqslant \alpha / p$ and the result follows from the maximum principle.

For any $f \in S(p)$, let $z_{1}, \ldots, z_{p}$ be the zeros of $f(z)$, let $r_{i}=\left|z_{i}\right|, R_{M}=$ $\max \left\{r_{i}\right\}, R_{m}=\min \left\{r_{i} \mid r_{i} \neq 0\right\}$, and let $r=|z|$. We also assume that the constant $A$ of Theorem 2.1 equals 1 .

THEOREM 2.2. Let $S_{1}\left(p, \alpha ; z_{1}, \ldots, z_{p}\right)$ denote the subclass of $S_{1}(p, \alpha)$ of functions with zeros at $z_{1}, \ldots, z_{p}$. Then the extreme points of the closed convex hull of $S_{1}\left(p, \alpha ; z_{1}, \ldots, z_{p}\right)$ are precisely the functions of the form

$$
f(z)=\prod_{j=1}^{p} \Psi\left(z_{1} z_{j}\right) z^{p}(1-x z)^{\alpha-2 p}, \quad|x|=1 .
$$

Proof. It follows from (2.2) and the compactness of $S(\alpha / p)$ that $S_{1}\left(p, \alpha ; z_{1}, \ldots, z_{p}\right)$ is compact. For $z_{1}, \ldots, z_{p}$ fixed the mapping

$$
T:[g(z)]^{p} \rightarrow \prod_{j=1}^{p} \Psi\left(z_{1} z_{j}\right)[g(z)]^{p}
$$

is a linear homomorphism; thus it suffices to find the extreme points of $\left\{[g(z)]^{p} \mid g \in S(\alpha / p)\right\}$. Since $p>0$, the argument in [3] applies and we are done.

Corollary 2.3. Let $f(z)=a_{0}+\ldots \in S_{1}\left(p, \alpha ; z_{1}, \ldots, z_{p}\right)$. Then

(i) $|f(z)| \leqslant \Pi_{j=1}^{p}\left(r+r_{j}\right)\left(1+r_{j} r\right)(1-r)^{\alpha-2 p},|z|<1$,

(ii) $|f(z)| \geqslant \prod_{j=1}^{p}\left(r-r_{j}\right)\left(1-r_{j} r\right)(1+r)^{\alpha-2 p},|z|>R_{M}$,

(iii) $|f(z)| \geqslant \prod_{j=1}^{p}\left(r_{j}-r\right)\left(1-r_{j} r\right)(1+r)^{\alpha-2 p}, \quad|z|<R_{m}$,

(iv) $\left|a_{n}\right| \leqslant A_{n}$, where $A_{n}$ is the coefficient of $z^{n}$ in

$$
F(z)=\prod_{j=1}^{p}\left(z+r_{j}\right)\left(1+r_{j} z\right)(1-z)^{\alpha-2 p},
$$

(v) $\left|f^{(k)}(z)\right| \leqslant F^{(k)}(r), k=1,2, \ldots$ 
We note that it is possible to obtain sharp upper and lower bounds for $\operatorname{Re}\left(z f^{\prime}(z) / f(z)\right)$ using the estimates in [6], but we do not state them here.

The problem of determining $\max \left|a_{n}\right|$ when $\left|a_{1}\right|, \ldots,\left|a_{p}\right|$ are fixed was first studied for the class $S(p)$ by Goodman [4]. We are only able to obtain a partial result.

LEMMA 2.4. Let $f(z)=\left(z-z_{0}\right)\left(1-\bar{z}_{0} z\right) \cdot z^{-1}\left(z+\sum_{n=2}^{\infty} b_{n} z^{n}\right)^{p}$. Then if $f(z)=\Sigma_{n=p-1}^{\infty} a_{n} z^{n}$,

$$
a_{p+1}=\left[p b_{3}-\frac{p(p+1)}{2} b_{2}^{2}+\frac{\overline{z_{0}}}{z_{0}}\right] a_{p-1}+p b_{2} a_{p} .
$$

Proof. Let $\Sigma_{1}^{\infty} c_{n} z^{n}=\left(\Sigma_{1}^{\infty} b_{n} z^{n}\right)^{p}, b_{1}=1$. Then

$$
\begin{aligned}
f(z)= & -z_{0} c_{p} z^{p-1}+\left[-z_{0} c_{p+1}+\left(1+\left|z_{0}\right|^{2}\right) c_{p}\right] z^{p} \\
& +\left[-z_{0} c_{p+2}+\left(1+\left|z_{0}\right|^{2}\right) c_{p+1}-\bar{z}_{0} c_{p}\right] z^{p+1}+\cdots .
\end{aligned}
$$

Comparing coefficients, we have

$$
\begin{aligned}
& a_{p-1}=-z_{0} c_{p}, \quad a_{p}=-z_{0} c_{p+1}+\left(1+\left|z_{0}\right|^{2}\right) c_{p}, \\
& a_{p+1}=-z_{0} c_{p+2}+\left(1+\left|z_{0}\right|^{2}\right) c_{p+1}-\bar{z}_{0} c_{p} .
\end{aligned}
$$

An easy calculation yields

$$
a_{p+1}=\frac{c_{p+1}}{c_{p}} a_{p}+\left[\frac{c_{p+2}}{c_{p}}-\left(\frac{c_{p+1}}{c_{p}}\right)^{2}+\frac{\overline{z_{0}}}{z_{0}}\right] a_{p-1} .
$$

Since $c_{p}=1, c_{p+1}=p b_{2}$, and $c_{p+2}=p(p-1) b_{2}^{2} / 2+p b_{3}$, substitution of (2.5) yields (2.4).

THEOREM 2.5. Let $f(z)=a_{p-1} z^{p-1}+a_{p} z^{p}+\ldots \in S_{1}(p, \alpha)$ with $p \geqslant$ $2,\left(2 p^{2}-3 p-\left(5 p^{2}-4 p\right)^{1 / 2}\right) / 2(p-1) \geqslant \alpha \geqslant 0, a_{p}$ real. Then

$$
\left|a_{p+1}\right| \leqslant\left[2(p-\alpha)^{2}-(p-\alpha)-1\right]\left|a_{p-1}\right|+(p-\alpha)\left|a_{p}\right| \text {. }
$$

Proof. It follows from Theorem 2.1 that $f(z)$ has a single real zero $z_{0}$ not at the origin and that $g(z)=z+\sum_{n=2}^{\infty} b_{n} z^{n}$ has real coefficients. From (2.4) we have

$$
\left|a_{p+1}\right| \leqslant\left(1+p\left|b_{3}-\frac{(p+1)}{2} b_{2}^{2}\right|\right)\left|a_{p-1}\right|+p\left|b_{2}\right|\left|a_{p}\right| .
$$


For $\alpha / p \leqslant 1-1 / p$, it follows from a result of Keogh and Merkes [7] that $\left|b_{3}-(p+1) b_{2}^{2} / 2\right| \leqslant(1-\alpha / p)(2 p-2 \alpha-1)$ and thus

$$
1+p\left(b_{3}-\frac{p+1}{2} b_{2}^{2}\right) \geqslant 1-(p-\alpha)(2 p-2 \alpha-1) .
$$

It remains to find an upper bound for the left-hand side of (2.6). It suffices to prove

$$
(p-\alpha)(2 p-2 \alpha-1)-1+p\left(\frac{p+1}{2}\right) b_{2}^{2} \geqslant 1+p b_{3},
$$

which is certainly true if

$$
(p-\alpha)(2 p-2 \alpha-1) \geqslant 2+p b_{3} .
$$

Since $\left|b_{3}\right| \leqslant(1-\alpha / p)(3-2 \alpha / p)[3],[11],(2.7)$ is certainly true if $(p-\alpha)(2 p-2 \alpha-1) \geqslant 2+(p-\alpha)(3-2 \alpha / p)$, or

$$
\alpha \leqslant\left(2 p^{2}-3 p-\left(5 p^{2}-4 p\right)^{1 / 2}\right) / 2(p-1) \text {. }
$$

This proves the theorem, since $\left|b_{2}\right| \leqslant(1-\alpha / p)[3],[11]$.

3. Let $S_{2}(p, \alpha)$ denote the subclass of $S(p)$ consisting of all functions $f(z)$ for which

$$
\lim \sup \min \operatorname{Re}\left[\alpha \frac{1+z f^{\prime \prime}(z)}{f^{\prime}(z)}+(1-\alpha) \frac{z f^{\prime}(z)}{f(z)}\right] \geqslant 0 .
$$

The class $S_{2}(p, \alpha)$ is the analog of the class $C(\alpha)$ of $\alpha$-convex functions defined by Mocanu [10]. A short calculation gives

THEOREM 3.1. Let $f(z) \in S_{2}(p, \alpha)$. Then $f(z)=\Pi_{j=1}^{p} \Psi\left(z, z_{j}\right)[g(z)]^{p}$, where $g(z) \in C(\alpha / p)$.

We cannot state an analogue of Theorem 2.2 since as yet the extreme points of $C(\beta)$ are not known for all $\beta$. However, we can obtain the results of Corollary 2.3 .

THEOREM 3.2. Let $f(z)=a_{0}+\ldots \in S_{2}(p, \alpha), \alpha>0$. Then:

(i) $|f(z)| \leqslant \prod_{j=1}^{p}\left(r+r_{j}\right)\left(1+r_{j} r\right) r^{-p}\left[\frac{p}{\alpha} \int_{0}^{r}(1-t)^{-p / \alpha} t^{p / \alpha-1} d t\right]^{\alpha}, \quad|z|<1$,

(ii) $|f(z)| \geqslant \prod_{j=1}^{p}\left(r-r_{j}\right)\left(1-r_{j} r\right) r^{-p}\left[\frac{p}{\alpha} \int_{0}^{r}(1+t)^{-p / \alpha} t^{p / \alpha-1} d t\right]^{\alpha}, \quad|z|>R_{M}$,

(iii) $|f(z)| \geqslant \prod_{j=1}^{p}\left(r_{j}-r\right)\left(1-r_{j} r\right) r^{-p}\left[\frac{p}{\alpha} \int_{0}^{r}(1+t)^{-p / \alpha} t^{p / \alpha-1} d t\right]^{\alpha}, \quad|z|<R_{m}$, 
(iv) $\left|a_{n}\right| \leqslant A_{n}$; where

$$
F(z)=\prod_{j=1}^{p} \Psi\left(z,-r_{j}\right)\left[\frac{p}{\alpha} \int_{0}^{z}(1-t)^{-p / \alpha} t^{p / \alpha-1} d t\right]^{\alpha}=\sum_{n=0}^{\infty} A_{n} z^{n},
$$

(v) $\left|f^{(k)}(z)\right| \leqslant F^{(k)}(r), k=1,2, \ldots$

Proof. Inequalities (i), (ii) and (iii) follow from Theorem 3.1 and the distortion theorem for $\alpha$-convex functions (see Miller [9]). Statements (iv) and (v) follow from the recent result of P. Kulshrestha [8].

We mention without proof that a result similar to Theorem 2.5 holds for $S_{2}(p, \alpha)$ using the technique of Theorem 2.5 and a result of J. Syzmal [12].

4. Let $S_{3}(p, \alpha), 0 \leqslant \alpha \leqslant 1$, denote the subclass of $S(p)$ consisting of all functions $f$ for which

$$
\text { lim sup } \max \left|\arg \left(z f^{\prime}(z) / f(z)\right)\right| \leqslant \alpha \pi / 2 .
$$

This extends the class $S^{*}(\alpha)$ of strongly starlike functions of order $\alpha$ defined by D. Brannan and W. Kirwan [2]. Note that a single valued branch of $\arg \left(z f^{\prime}(z) / f(z)\right)$ can be defined in some annulus $\rho<|z|<1$.

THEOREM 4.1. Let $f(z) \in S_{3}(p, \alpha)$. Then there is a function $g(z) \in S^{*}(\alpha)$ such that $f(z)=\Pi_{j=1}^{p} \Psi\left(z, z_{j}\right) g(z)^{p}$.

Proof. This follows from the equation (2.3) since $\operatorname{Re}\left(z \Psi^{\prime}\left(z, z_{j}\right) / \Psi\left(z, z_{j}\right)\right)$ $=0$ on $|z|=1$.

CoRollary 4.2. If $f \in S_{3}(p, \alpha)$, then $f$ is bounded in $U$.

Proof. This follows from [2, Theorem 2.1] and the previous theorem.

LEMmA 4.3. Let $g(z)=z+b_{2} z^{2}+\ldots \in S^{*}(\alpha)$. Then if either $\lambda \geqslant 3 / 4$ or $3 / 4-1 / 4 \alpha \geqslant \lambda,\left|b_{3}-\lambda_{2}^{2}\right| \leqslant\left|3 \alpha^{2}-4 \lambda \alpha^{2}\right|$, and this result is sharp.

Proof. Using the notation of [1, Theorem 2.1], we have

$$
b_{3}-\lambda b_{2}^{2}=\frac{\alpha}{2}\left[p_{2}+\frac{3 \alpha-1-4 \lambda \alpha}{2} p_{1}^{2}\right],
$$

where $P(z)=1+p_{1} z+p_{2} z^{2}+\ldots$ has $\operatorname{Re} P(z)>0$ in $U$. We have

$$
\begin{aligned}
b_{3}-\lambda b_{2}^{2}= & \frac{\alpha}{2} \cdot 2 \int_{0}^{2 \pi} e^{-2 i \theta} d \mu(\theta) \\
& +\frac{\alpha}{2}\left(\frac{3 \alpha-1-4 \lambda \alpha}{2}\right)\left(2 \int_{0}^{2 \pi} e^{-i \theta} d \mu(\theta)\right)^{2},
\end{aligned}
$$

where $\mu(\theta)$ is an increasing function on $[0,2 \pi]$ with $\mu(2 \pi)-\mu(0)=1$. Hence 


$$
\begin{aligned}
\frac{2}{\alpha} \operatorname{Re}\left(b_{3}-\lambda b_{2}^{2}\right)= & 2 \int_{0}^{2 \pi} \cos \theta d \mu(\theta) \\
& +(6 \alpha-2-8 \lambda \alpha)\left[\left(\int_{0}^{2 \pi} \cos \theta d \mu(\theta)\right)^{2}-\left(\int_{0}^{2 \pi} \sin \theta d \mu(\theta)\right)^{2}\right] .
\end{aligned}
$$

Suppose first that $6 \alpha-2-8 \lambda \alpha \geqslant 0$. Then

$$
\begin{aligned}
\frac{2}{\alpha} \operatorname{Re}\left(b_{3}-\lambda b_{2}^{2}\right) & \leqslant 2 \int_{0}^{2 \pi} \cos 2 \theta d \mu(\theta)+(6 \alpha-2-8 \lambda \alpha)\left(\int_{0}^{2 \pi} \cos \theta d \mu(\theta)\right)^{2} \\
& \leqslant 4 \int_{0}^{2 \pi} \cos ^{2} \theta d \mu(\theta)-2+(6 \alpha-2-8 \lambda \alpha) \int_{0}^{2 \pi} \cos ^{2} \theta d \mu(\theta) \\
& \leqslant 6 \alpha-8 \lambda \alpha,
\end{aligned}
$$

where we have used Jensen's inequality and the estimate $\int_{0}^{2 \pi} \cos ^{2} \theta d \mu(\theta) \leqslant 1$.

The case $6 \alpha-2-8 \lambda \alpha<0$ is treated in a similar manner.

To show that the inequality is sharp, we consider the function $g(z)$ defined by

$$
z g^{\prime}(z) / g(z)=((1+z) /(1-z))^{\alpha}
$$

for which $a_{2}=2 \alpha, b_{3}=3 \alpha^{2}$.

THEOREM 4.4. Let $f(z)=a_{p-1} z^{p-1}+a_{p} z^{p}+$ in $\in S_{3}(p, \alpha)$ and suppose each $a_{n}$ is real. If $p \geqslant 3$ and $\alpha \geqslant \min \left(1 / 3,\left(p^{2}-2 p\right)^{-1 / 2}\right)$,

$$
\left|a_{p+1}\right| \leqslant\left(2 p^{2} \alpha^{2}-p \alpha^{2}-1\right)\left|a_{p-1}\right|+2 \alpha p\left|a_{p}\right|
$$

and this result is sharp.

Proof. By Lemma 2.4, it is sufficient to show that

$$
\left|1+p b_{3}-p\left(\frac{p+1}{2}\right) b_{2}^{2}\right| \leqslant 2 p^{2} \alpha^{2}-p \alpha^{2}-1,
$$

since $\left|b_{2}\right| \leqslant 2 \alpha[1]$. By Lemma 2.3, with $\lambda=(p+1) / 2$,

$$
1+p b_{3}-p((p+1) / 2) b_{2}^{2} \geqslant 1+p \alpha^{2}-2 p^{2} \alpha^{2}
$$

and hence it suffices to show

$$
1+p b_{3} \leqslant 2 p^{2} \alpha^{2}-p \alpha^{2}-1 \text {. }
$$

Since $\left|b_{3}\right| \leqslant 3 \alpha^{2}$ if $\alpha \geqslant 1 / 3,(4.2)$ follows if $1+3 p \alpha^{2} \leqslant 2 p^{2} \alpha^{2}-p \alpha^{2}-1$, which is certainly true if $p \geqslant 3$.

The sharpness of the result follows from the fact that the function $g(z)$ defined by (4.1) simultaneously maximizes $\left|b_{2}\right|$ and $\left|b_{3}-(p+1) b_{2}^{2} / 2\right|$. 
Notes. 1. If $p \geqslant 4$, the result holds for all $\alpha \geqslant 1 / 3$.

2. For $0<\alpha \leqslant 1 / 3$, a similar (but not sharp) result holds for $p>$ $\left[\alpha^{2}+\alpha+\left(17 \alpha^{4}+2 \alpha^{3}+\alpha^{2}\right)\right]\left(4 \alpha^{2}\right)^{-1}$.

\section{REFERENCES}

1. D. A. Brannan, J. Clunie and W. E. Kirwan, Coefficient estimates for a class of star-like functions, Canad. J. Math. 22 (1970), 476-485. MR 41 \#5614.

2. D. Brannan and $\mathrm{W}$. Kirwan, On some classes of bounded univalent functions, $\mathrm{J}$. London Math. Soc. (2) 1 (1969), 431-443. MR 40 \#4439.

3. L. Brickman, D. Hallenbeck, T. MacGregor and D. Wilken, Convex hulls and extreme points of families of starlike and convex mappings, Trans. Amer. Math. Soc. 185 (1973), 413-428.

4. A. W. Goodman, On the Schwarz-Christoffel transformation and p-valent functions, Trans. Amer. Math. Soc. 68 (1950), 204-223. MR 11, 508.

5. J. A. Hummel, Multivalent starlike functions, J. Analyse Math. 18 (1967), 133160. MR $35 \# 359$.

6. - Extremal properties of weakly starlike p-valent functions, Trans. Amer. Math. Soc. 130 (1968), 544-551. MR 36 \#5332.

7. F. R. Keogh and E. P. Merkes, A coefficient inequality for certain classes of analytic functions, Proc. Amer. Math. Soc. 20 (1969), 8-12. MR 38 \#1249.

8. P. Kulshrestha, Coefficients for alpha-convex univalent functions, Bull. Amer. Math. Soc. 80 (1974), 341-342.

9. S. Miller, Distortion properties of alpha-starlike functions, Proc. Amer. Math. Soc. 38 (1973), 311-318. MR 46 \#9324.

10. P. T. Mocanu, Une propriété de convexité généralisée dans la théorie de la représentation conforme, Mathematica (Cluj) 11 (34) (1969), 127-133. MR 42 \#7881.

11. M. S. Robertson, On the theory of univalent functions, Ann. of Math. (2) 37 (1936), 374-408.

12. J. Syzmal, Some remarks on coefficients inequality for $\alpha$-convex functions, Bull. Acad. Polon. Sci. Sér. Sci. Math. Astronom. Phys. 20 (1972), 917-919.

DEPARTMENT OF MATHEMATICS, HOWARD UNIVERSITY, WASHINGTON, D. C. 20001 